\title{
AdAPTIVE ELECTROMAGNETIC ENERGY HARVESTER FOR MOBILE DEVICES
}

\author{
Haziq Kamal \\ Robotics and Autonomous Systems \\ CSIRO Data61 \\ QCAT, 1 Technology Court, Pullenvale \\ QLD 4069, Australia \\ muhammad.kamalarifin@uq.net.au
}

\author{
Peyman Moghadam* \\ Robotics and Autonomous Systems \\ CSIRO Data61 \\ QCAT, 1 Technology Court, Pullenvale \\ QLD 4069, Australia \\ peyman.moghadam@csiro.au
}

\begin{abstract}
Advances in design and development of light-weight and low power wearable and mobile devices open up the possibility of lifetime extension of these devices from ambient sources through energy harvesting devices as opposed to periodically recharge the batteries. The most commonly available ambient energy source for mobile devices is Kinetic energy harvesters $(\mathrm{KEH})$. The major drawback of the energy harvesters is limited effectiveness of harvesting mechanism near a fixed resonant frequency. It is difficult to harvest a reliable amount of energy from every forms of device motions with different excitation frequencies. To overcome this drawback, in this paper we propose an adaptive electromagnetic energy harvester which utilises spring characteristics to adapt its resonant frequency to match the ambient excitation frequency. This paper presents a prototype design and analysis of an adaptive electromagnetic energy harvester both in simulation and real. The harvester has tested using a specially designed experimental setup and compared with numerical simulations. The proposed solution generates 3.5 times higher maximum power over the default power output and 2.4 times higher maximum frequency compared to a fixed resonant frequency electromagnetic energy harvester.
\end{abstract}

Keywords mobile devices · kinetic energy harvester - electromagnetic energy harvester · nonlinear spring characteristic $\cdot$ resonant frequency $\cdot$ adaptive energy harvester $\cdot$ mobile robots

\section{INTRODUCTION}

Over the last decade, wearable and mobile devices have received increasing demand for a large variety of applications including healthcare [1], sports, security [2], entertainment [3], military, internet of things (IoT) [4] [5, 6, 7] and robotics [8]. One major challenge of these ubiquitous devices is their limited lifetime. They are typically powered by batteries which needs to periodically recharge or replace. To achieve lifetime extension, energy harvesting from ambient sources is a promising solution towards self-powered wearable, mobile devices and mobile robots [9, 10, 11].

The most commonly available ambient energy sources for wearable devices are infrared radiant energy [12 13], solar energy [14] and kinetic energy (motion and vibration) [14] 15]. Kinetic energy harvesters (KEH) are particularly attractive energy sources for mobile technologies since movement or vibrations are considered to be the most abundant. Kinetic energy harvesters convert the movements into electrical energy. The main kinetic energy harvesters are based on piezoelectric [15, 16], electromagnetic [17, 18] or electrostatic [19] energy conversion mechanisms.

The major drawback of the kinetic energy harvesters is limited effectiveness of harvesting mechanism near a fixed resonant frequency [20]. The resonant frequency of most of the kinetic energy harvesters are fixed and therefore when the ambient excitation frequency deviates from the mechanical resonant frequency the generated power reduces significantly [21]. Human body movements in particular are not consistent and it is difficult to harvest a reliable amount of energy from every forms of human motions with different excitation frequencies. The same limitation is applicable to self-powered robotic platforms where the resonant frequency of mobile robots changes as they move from one type of terrains to others [22, 23, 24].

To overcome this limitation, several previous studies have investigated different approaches such as broadband energy harvesting, resonance tuning and hybrid techniques. This papers belongs to the resonance tuning category [25, 26].

\footnotetext{
${ }^{*}$ Corresponding author: Dr. Peyman Moghadam
} 
Several previous studies have investigated the development of resonance tuning methods for kinetic energy harvesting [27, 28, 29]. A common approach to broaden the effective frequency range is to add more degrees of freedom such as multiple oscillators or multiple masses on single cantilever beam. These methods increase the number of resonance points in the system [28]. However, these methods require more complex designs and increase in size and weights.

This paper proposes an adaptive, low-frequency kinetic energy harvester based on the linear electromagnetic energy technique. The main contribution of this paper is the design concept of autonomously adapt the resonant frequency of an electromagnetic energy harvester by utilizing spring characteristics to match the ambient excitation frequency. The proposed electromagnetic energy harvester could match its resonant frequency to ambient excitation frequency by changing stiffness of a secondary spring by applying different forces.

The rest of this paper is organized as follows. Section 2 gives an overview of the most common kinetic energy harvesters. In Section 3 we present mathematical model for electromagnetic energy harvesters which is used for our simulation. Section 4 describes the design of the prototype adaptive kinetic energy harvester and the experimental setup for testing the prototype. Section 5 , the measured experimental results are analyzed and compared with the simulation results of the mathematical model implemented. Finally, Section 6 concludes the paper with potential future directions.

\section{Related Work}

Kinetic energy harvester can be generally classified into two main types which include piezoelectric and electromagnetic energy harvester. This section will layout the basics and state of art of these two types of generators.

\subsection{Piezoelectric Energy Harvester}

Piezoelectric energy harvester uses material such as a laminate of polyvinylidene fluoride (PVDF) or lead zirconate titanate (PZT) that will convert any stress or strain applied to it to electrical energy. This occurs by separating the charges (positive and negative) at opposite locations thus creating a potential difference that will drive the electrons which is equivalent to an induced current.

Piezoelectric energy harvesters can be classified into two spectrum of frequencies; low frequency $(0 \mathrm{~Hz}$ $20 \mathrm{~Hz}$ ) which is suitable to harvest energy from human or animal movements and high frequency (higher than $20 \mathrm{~Hz}$ ), vibration from any machines or structures. The first energy-harvesting based on human walking have been developed where an elongated hexagon-shaped sneaker insole is made up of PVDF [30] as shown in Figure 1a Piezoelectric energy harvester has been also integrated in footwear to convert pressure variations to energy from people walking [31].

Vibration based piezoelectric generator usually takes a form of a cantilever beam as shown in Figure $1 \mathrm{~b}$ Piezoelectric harvester with the shape of a cantilever beam also has been used to exploit energy from rotary motion [32] which has a big potential for any machines and robots since gears and rotating motors are widely used by them. The main disadvantage of piezoelectric energy harvesters is that they produce a very low current although the output voltage is high. The advantage of piezoelectric energy harvesters is flexibility, easy integration and fabrication into apparels.

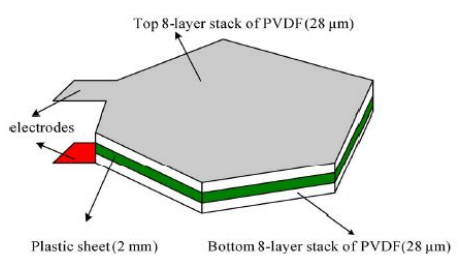

(a)

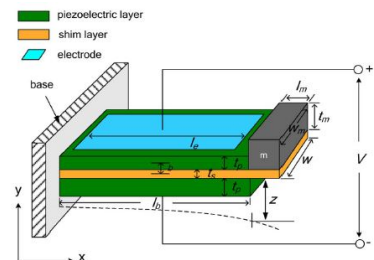

(b)

Figure 1: Piezoelectric energy harvesters. (a) Layout of PVDF sneaker insole [30]. (b) Vibration-based cantilever piezoelectric energy harvester [33].

\subsection{Electromagnetic Energy Harvester}

Electromagnetic energy harvester is based on Faraday's Law of induction. Rate of change in magnetic field of a coil causes a voltage to be induced. This type of harvester consists of a permanent magnet and a coil. Two main categories for an electromagnetic energy harvester are resonant and rotational generator.

The focus in this paper is the resonant electromagnetic energy harvester which comprises of a permanent magnet, a coil and a spring as shown in Figure 2a [26]. Such configuration is common for an electromagnetic energy harvester such as a double permanent magnet vibration power generator [25] that has been built practically for smart hip prosthesis as shown in Figure $2 \mathrm{~b}$ This type of system has a fixed resonant frequency that matches with a human movements such as walking and slow running. Electromagnetic energy harvesters are very suitable for wearable and robotic devices due to the high current outputs. 
However, this type of electromagnetic energy harvester only has a fixed resonant frequency. This is not suitable for a platform that can vibrate in different frequencies. Robot movements are not consistent and it is difficult to harvest a reliable amount of energy from every forms of robot motions. To overcome these limitation, recent studies explores nonlinear techniques to obtain broadband energy harvesters [34, 35]. Mann et al. [34] utilizes magnetic levitation-based energy harvesting which works similar to spring characteristics by two outer magnets that they are mechanically attached to a threaded support and a suspending center magnet. However, adding magnet will increase the weight of the harvester drastically which is not practical in mobile devices.

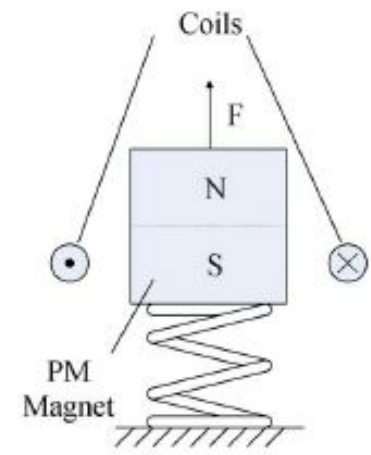

(a)

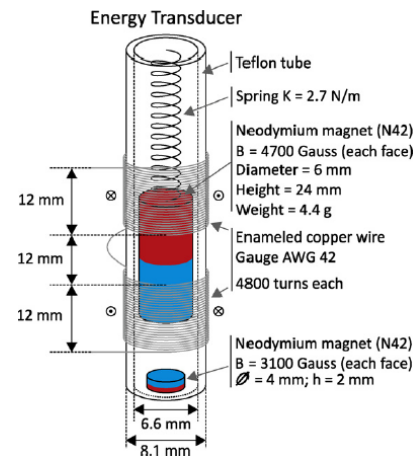

(b)

Figure 2: Electromagnetic energy harvesters. (a) Basic configuration for resonant electromagnetic energy harvester [26]. (b) Double permanent magnet vibration power generator for smart hip prosthesis [25].

\section{BACKGROUND}

In this section basic principle of electromagnetic energy harvester used for mathematical modeling is discussed.

\subsection{Principle of Electromagnetic Energy Harvester}

A generic resonant electromagnetic energy harvester can be modeled by a spring mass system with a continuous force acting upon the system as shown in Figure 3 [36]. Such model can be represented as:

$$
M \ddot{y}+C \dot{y}+K y+F_{e}=-M \ddot{u_{0}}
$$

where $y$ is the displacement of mass, $M$ is the effective mass of the system, $K$ is the total spring constant of the system and $C$ is the mechanical damping of mass $M$. The right-hand side of the equation is the continuous force which is being applied to the system. Backward coupling force, denoted by $F_{e}$, is also present in the equation above due to the opposing magnetic field that comes from the current flowing through the coil, which is given by

$$
F_{e}=\theta_{e} I_{R}
$$

where $I_{R}$ is the current flowing through the resistor $R_{L}$, and $\theta_{e}$ is the electromagnetic coupling coefficient which can be further expressed as

$$
\theta_{e}=B_{1} l
$$

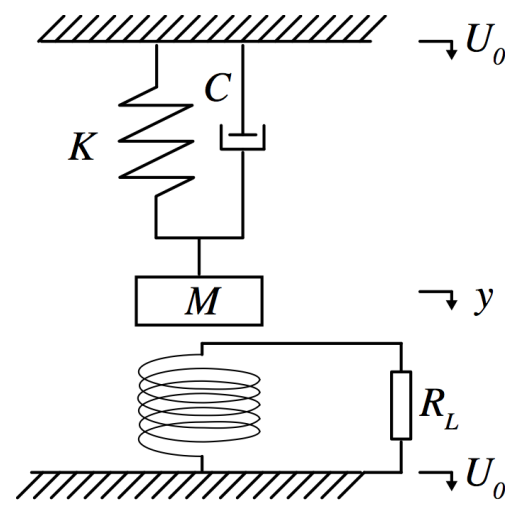

Figure 3: Diagram of a generic resonant electromagnetic harvester. 


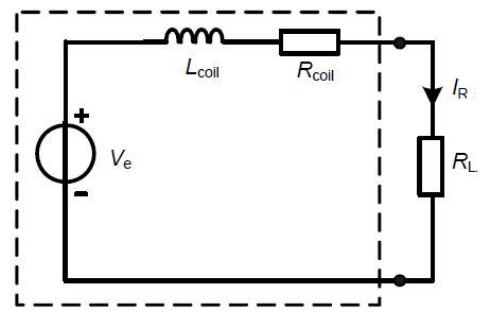

Figure 4: Equivalent electrical model of electromagnetic energy harvester [36].

where $B_{1}$ is the average magnetic flux density and $l$ is the total length of the coil.

A simplified electrical model which is equivalent to an electromagnetic harvester is illustrated in Figure 4 [36]. The model consists of a voltage source $V_{e}$ connected in series with an internal inductance $L_{\text {coil }}$, the coil resistance $R_{\text {coil }}$ and also the resistor $R_{L}$ at the output terminal.

By using Kirchoff's voltage law, the equation that governs the induction of voltage from electromagnetic harvester can be obtained as

$$
-V_{e}+L_{\text {coil }} \dot{I_{R}}+\left(R_{\text {coil }}+R_{L}\right) I_{R}=0
$$

where the voltage $V_{e}$ is proportional to the velocity and can be expressed as $V_{e}=\theta_{e} \dot{y}$.

Substituting Equations (2) and (3) into (1) and combining Equations (1) and (4) result in this overall equations:

$$
\left\{\begin{array}{l}
M \ddot{y}+C \dot{y}+K y+\theta_{e} I_{R}=-M \ddot{u_{0}} \\
-\theta_{e} \dot{y}+L_{\text {coil }} \dot{I_{R}}+\left(R_{\text {coil }}+R_{L}\right) I_{R}=0
\end{array}\right.
$$

Mechanical damping $C$, in the expression above can also be written as

$$
C=2 M \zeta \omega_{0}
$$

where $\zeta$ is the damping ratio of springs and resonant angular frequency $\omega_{0}$, can be expressed as

$$
\omega_{0}=\sqrt{K / M}
$$

By substituting Equation (6) and (7) and then applying Laplace transform to Equation (5), the end result for power being produced is as follows

$$
\begin{gathered}
\left|\frac{\hat{P_{R}}}{\left(\omega^{2} \hat{u_{0}}\right)^{2}}\right| \rightarrow \frac{M\left(\frac{\omega}{\omega_{0}}\right)^{2} k_{e}^{2} \omega_{e}}{\omega_{0}\left(1+R_{\text {coil }} / R_{L}\right)} \times \\
\left|\frac{1}{\left.\left.\left.\left(1-\left(\frac{\omega}{\omega_{0}}\right)^{2}+j 2 \zeta \frac{\omega}{\omega_{0}}\right)\right)\left(j \frac{\omega}{\omega_{0}}+\omega_{e}\right)+j k_{e}^{2} \frac{\omega}{\omega_{0}}\right)\right)}\right|^{2}
\end{gathered}
$$

where $\mathrm{j}$ is the imaginary unit and $\omega$ is the angular frequency of vibration that are obtained through Laplace transform which derives from $s=j \omega$ and the dimensionless parameters are

$$
\begin{gathered}
k_{e}^{2}=\frac{\theta_{e}^{2}}{L_{\text {coil }} K} \\
\omega_{e}=\frac{R_{\text {coil }}+R_{L}}{\omega_{0} L_{\text {coil }}}
\end{gathered}
$$

Based on Equation (8) power output is optimum when $\frac{\omega}{\omega_{0}}$ is close to one. Any deviation of the resonant angular frequency $\omega_{0}$ from the excitation angular frequency $\omega$ results in significant power output reduction.

\section{MATERIAL AND METHODS}

This section describes the design of our proposed adaptive, low-frequency kinetic energy harvester. The proposed electromagnetic energy harvester has a unique advantage where it can sense ambient frequency and adapt its resonant frequency using spring characteristic.

\subsection{Adaptable Resonant Frequency of Electromagnetic Energy Harvester}

An electromagnetic harvester uses an inductive spring mass system for converting mechanical energy to electrical as shown in Figure 5 In this paper we are using an off-the-shelf electromagnetic energy harvester (nPower PEG) that has a resonant frequency fixed at $2-3 \mathrm{~Hz}$ which is tuned for human walking and slow running movements. Such energy harvester can only operate efficiently whenever the frequency of the external movement matches the 


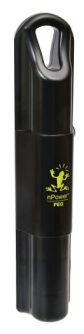

(a)

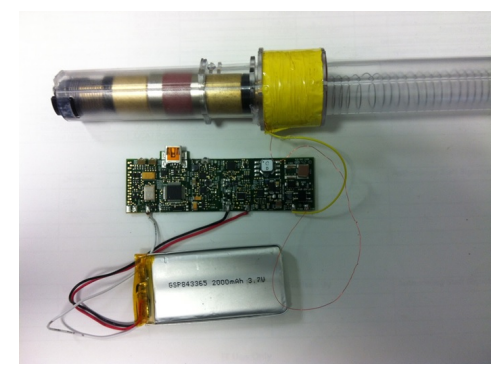

(b)

Figure 5: An off-the-shelf nPower PEG consists of an electromagnetic harvester, a circuit that converts alternating current to a direct current with $3.7 \mathrm{~V}$ peak and a battery storage.

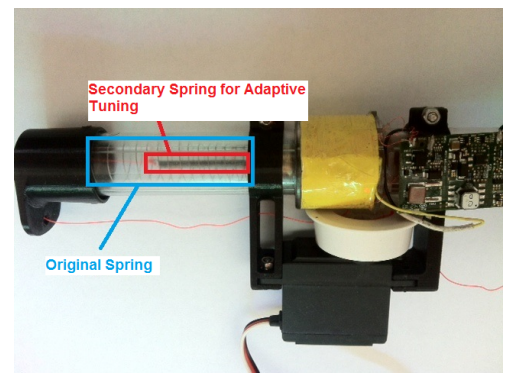

Figure 6: A prototype setup of the secondary spring inside the proposed prototype.

resonant frequency of the spring mass system. If the external frequency varies slightly compared to the resonant frequency, the power generated drops significantly by $80 \%$.

In order to tune the resonant frequency of a spring mass system, there are two possible parameters that can be varied based on Equation (7) which are mass of the system $M$, and total spring constant $K$. Changing $M$ is not possible due to the weight and space constraints for mobile devices. The approach proposes in this paper is by changing the total spring constant, $K$ by using spring characteristics. Spring constant or stiffness of a spring can be manipulated by applying different force to a spring. This paper uses the concept of hardening spring based on principle of spring constant.

The proposed system consists of two sets of springs, original passive spring and secondary linear spring attached inside the original passive spring where its spring constant is changed by applying different pulling forces by using a motor and mechanical locking clamp. The proposed prototype is shown in Figure 6

Total spring constant of the overall system can be written as

$$
K=k_{1}+k_{2}(t)
$$

where $k_{1}$ is the original passive spring constant of nPower PEG and $k_{2}(t)$ is the changing resonant frequency of the secondary spring. The total spring constant of the system is the summation of $k_{1}$ and $k_{2}(t)$ since the original and secondary springs are in a parallel configuration as shown in Figure 6

\subsection{Data Acquisition and Processing Units}

The prototype was developed by using Arduino Uno as the main processor unit, 9 Degrees of Freedom (9DOF)Razor IMU as a sensor to measure acceleration valued and estimate the ambient frequency of the platform, SD Card to store data on board for analysis, motor and mechanical locking clamp to apply force to the secondary spring. Mechanical locking clamp is used to provide a passive tension in the spring without drawing current.

The 9DOF-Razor IMU consists of a gyroscope, accelerometer and a magnetometer. The data from magnetometer in 9DOF-Razor IMU was ignored since the magnetic field from the permanent magnet is interfering with the Earth's magnetic field that should be detected by the magnetometer. The data obtained from gyroscope and accelerometer has to be processed and calibrated to neglect the acceleration comes from gravity and to get the actual vertical acceleration.

The accelerometer reading is taken when the accelerometer is stationary and is assumed to be the gravity vector. Next, the angle in which the device is tilted should be estimated by using angular data obtained from gyroscope and integrate them as follows

$$
\text { gyroAngle }+=\frac{\text { gyroRate }}{\text { gyroSensitivity }} d t
$$




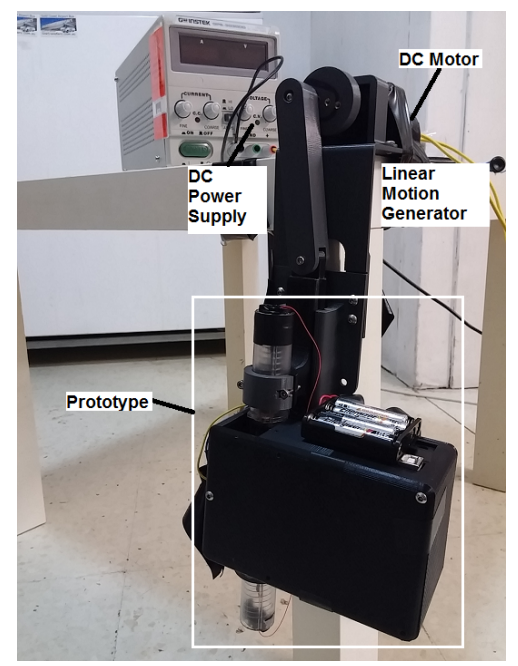

Figure 7: The overall experimental setup.

However, error builds up over time due to this integration and causes the data to drift. The complementary filter is used to compensate for the integration error. This is done by merging a large amount of gyroscope data (that has accumulated error, but not susceptible to gravity) with a small amount of accelerometer data (that has the problem of having gravity measured). By applying complementary filter, angle from gravity vector are obtained as

$$
\text { angle }=\alpha \times(\text { angle }+ \text { gyroAngle })+(1-\alpha) \times \text { accAngle }
$$

With this angle, the rotation of accelerometer data to the gravity plane is applied and the gravity is removed from the data. The output from 9DOF-Razor IMU which was the vertical acceleration was then being transmitted to Arduino Uno. The vertical acceleration was being stored until there were 256 points, which then will be used to estimate the excitation frequency of the system through Fast Fourier Transform (FFT).

The current frequency obtained from FFT can be used to adapt the resonant frequency of the spring mass system. By matching the resonant frequency with the current ambient excitation frequency, the power generated by the electromagnetic energy harvester is at the maximum value. Besides that, the voltage induced by electromagnetic energy harvester can be calculated using this formula

$$
V_{\text {induced }}=\frac{V_{A D C}}{1024} \times V_{\text {in }}
$$

where $V_{A D C}$ is the reading of Analogue to Digital Converter pins and $V_{i n}$ is the voltage input which is $5 \mathrm{~V}$.

\subsection{Experimental Setup}

Figure 7 shows the experimental setup for testing the prototype. The experimental setup consists of our prototype adaptive electromagnetic energy harvester device, linear motion generator and a DC power supply. We have designed and 3-D printed linear motion generator to provide repeatable mechanical excitation for the experimental setup (Figure 8). The linear motion generator uses a DC motor (HG42Kd2100) with a voltage rating up to $12 \mathrm{~V}$ and has a maximum angular speed of 500rpm. The linear motion generator converts rotational movement of the DC motor to linear vertical motion for our experiments. The maximum frequency that can be obtained is $8.33 \mathrm{~Hz}$ with a fixed peak oscillation of $1.5 \mathrm{~cm}$. To eliminate any possible vigorous vibrations, the prototype electromagnetic energy harvester device is firmly mounted on the linear motion generator and then they are secured to a table during the experiments (Figure 7). We use the AA batteries for powering the Arduino Uno to log performance data for further analysis.

\section{EXPERIMENTAL and SIMULATION RESULTS}

This section discusses the experimental and simulated results for the proposed adaptive, low-frequency KEH.

\subsection{Experimental results}

The linear motion generator is set to provide mechanical excitation with resonant ambient frequencies of $3 \mathrm{~Hz}$ to $7 \mathrm{~Hz}$. The ambient frequency then is estimated using the IMU data for 256 samples at each level of excitations. We accepted tolerance of less than $0.5 \mathrm{~Hz}$ as a good estimation of ambient excitation frequency based on IMU data. Then, we used the passive mechanical locking clamp and motor at four levels of stiffness to apply forces on the secondary spring where Level 1 receives the least pulling force and Level 4 has the highest. The 'Original' level of stiffness is when no pulling force is applied to the secondary spring. Each level of stiffness has been 


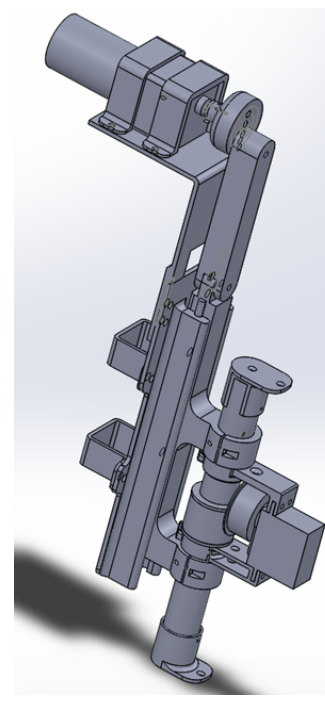

(a)

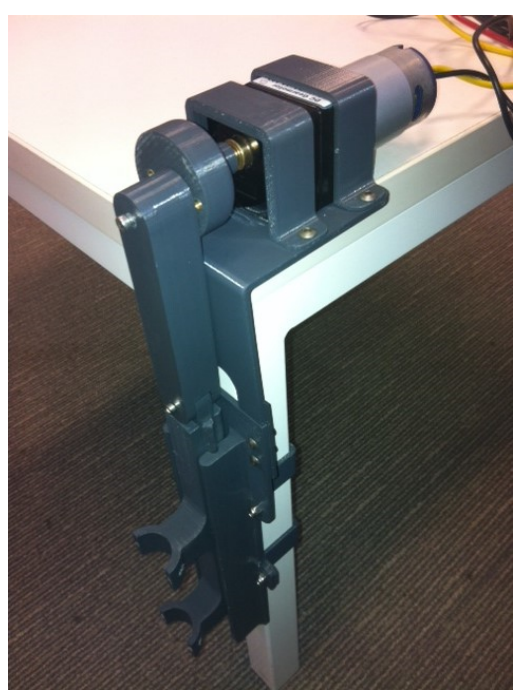

(b)

Figure 8: (a) 3D model of linear motion generator. (b) An image of the actual device.

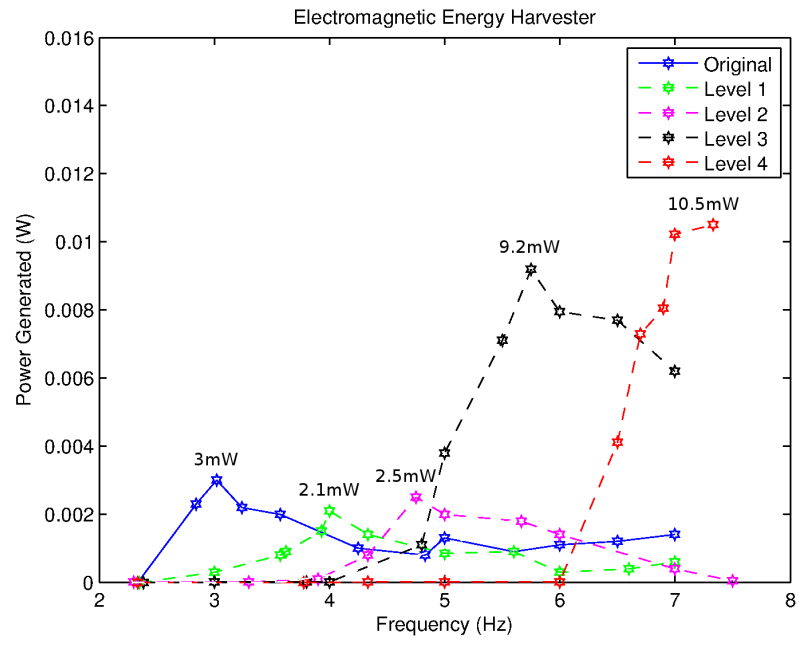

(a)

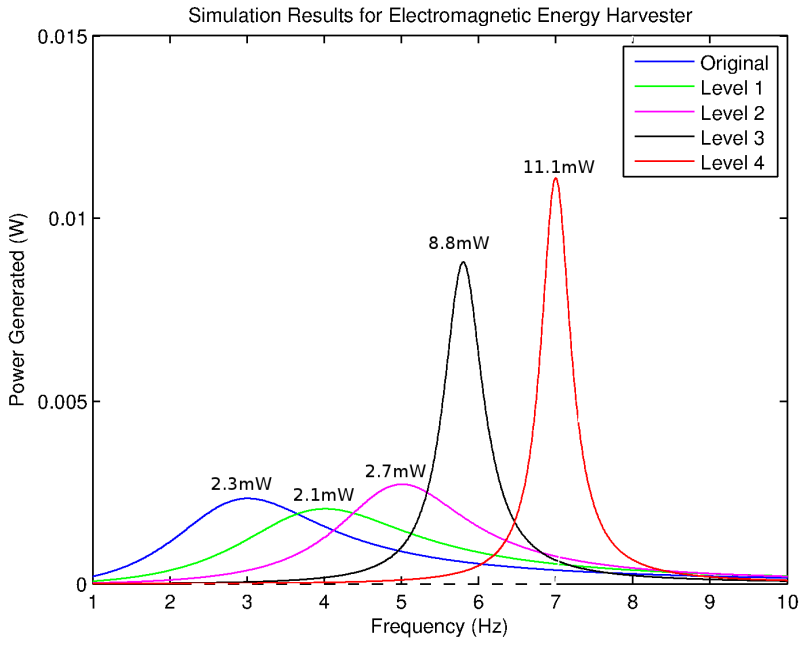

(b)

Figure 9: (a) Results from experiments using the prototype. (b) Simulation results of the mathematical model implemented using Matlab.

calibrated to achieve different $K$ values based on Equation 11 The output resonant frequency per level of stiffness is measured using the IMU on board for each spring $K$. These four level pre-selected for ranges of frequencies between $3 \mathrm{~Hz}$ to $7 \mathrm{~Hz}$ for this experiment. Based on spring characteristic, the spring constant increases as stiffness increases which increases the resonant frequency of spring mass system.

In Figure 9a the measured output power of prototype for different resonant frequencies based on different levels of spring stiffness is shown. We have used 4 levels of spring stiffness for the secondary spring and compared it to 'Original', the output power of the off-the-shelf nPower PEG. The prototype could produce the maximum power of $10.5 \mathrm{~mW}$ at frequency of $7.3 \mathrm{~Hz}$. The maximum output power is 3.5 times higher than the maximum power generated with the original nPower PEG product. The resonant frequency is 2.4 times higher $(7.3 \mathrm{~Hz})$ than the default frequency of the 'Original' device $(3 \mathrm{~Hz})$.

\subsection{Simulation}

For experimental validation, the numerical model based on Equation (8) was simulated using Matlab. We assumed the mass is under an excitation of $1 \mathrm{~m} / \mathrm{s}^{2}$ (RMS value). Table 1 summarizes parameters for magnet, coil and electrical circuit used in the simulation. One of the important parameters that needs calculation is the 
average magnetic flux density $B_{1}$ from Equation (3). Average magnetic flux density can be approximated by dividing the coil into $n$ sections [36],

$$
B_{1}=\frac{B_{r}}{2} \frac{1}{n} \sum_{i=1}^{n}\left[\frac{z_{i}+h_{m}}{\sqrt{\left(z_{i}+h_{m}\right)^{2}+r_{m}^{2}}}-\frac{z_{i}}{\sqrt{z_{i}^{2}+r_{m}^{2}}}\right]
$$

where $z_{i}$ is the distance from the magnet to the $i$ th cross section of the coil such that $z_{1}$ is the nearest distance from the magnet while $z_{n}$ is the farthest distance from the magnet. $B_{r}$ is residual flux density, $h_{m}$ is height of the magnet and $r_{m}$ is radius of the magnet.

Figure $9 \mathrm{~b}$ shows the results of simulation based on mathematical model are close to their measured output power (Figure $9 \mathrm{a}$ based on the experimental prototype device. The good agreement between experiments and simulations verifies the accuracy of the model and opens avenues for optimizing design of adaptive electromagnetic energy harvesters to achieve optimal power outputs.

\begin{tabular}{|c|c|}
\hline Parameter & Value \\
\hline Effective mass, $M(\mathrm{~g})$ & 86.9 \\
Radius of magnet, $r_{m}(\mathrm{~mm})$ & 9 \\
Height of magnet, $h_{m}(\mathrm{~mm})$ & 25 \\
Residual flux density, $B_{r}(\mathrm{~T})$ & 1.1 \\
Total length of the coil, $l(\mathrm{~m})$ & 261 \\
Inductance of the coil, $L_{\text {coil }}(\mathrm{mH})$ & 272.5 \\
Resistance of the coil, $R_{\text {coil }}(\mathrm{ohm})$ & 301.6 \\
Damping ratio of springs, $\zeta$ & 0.0035 \\
Load Resistance, $R_{L}(\mathrm{ohm})$ & 220 \\
\hline
\end{tabular}

Table 1: Parameters of magnet, coil and electrical circuit for the electromagnetic harvester. This parameters are used for the simulation results using Matlab.

\section{Conclusions}

In this paper, we have designed and developed a novel type of electromagnetic energy harvester that could adapt its resonant frequency to match the ambient excitation frequency. The prototype adaptively changes the stiffness of a nonlinear spring by applying different forces. The experimental results indicate that the proposed prototype resonant frequency increases by a factor of 2.4 , and its maximum power over a bandwidth increases by a factor of 3.5, as compared to an off-the-shelf electromagnetic energy harvester. We validated the experimental results with simulation results from the mathematical model. The good agreement between experiments and simulations verifies the accuracy of the model and opens avenues for optimizing design of adaptive electromagnetic energy harvesters to achieve optimal power outputs.

The main limitation of the proposed linear solution is the generated resonant frequency is not broadband as non-linear solutions. We will investigate coupling the propose solution with nonlinear techniques to broaden the frequency bandwidth of energy harvesting. Further design optimisation can be done in follow-up improvements to meet the form-factor and usability requirements for wearable or mobile devices. Finally, we will investigate coupling the proposed electromagnetic energy harvester with a piezoelectric cantilever beam to harvest higher frequency vibrations such as footfalls or robotic platforms moving on rough trains.

\section{Acknowledgment}

This work is supported by Commonwealth Scientific and Industrial Research Organization (CSIRO). Authors would like to thank Thong Nguyen for his contribution on experimental design. Authors would also like to thank Brett Wood, Ryan Steindl and Paul Flick for their helpful discussion and inputs in 3D modelling, design and experimental design.

\section{References}

[1] Peyman Moghadam. 3D medical thermography device. In Thermosense: Thermal Infrared Applications XXXVII, volume 9485, page 94851J. International Society for Optics and Photonics, 2015.

[2] Peyman Moghadam, Benjamin Evans, and Elliot Duff. SAGE: Semantic annotation of georeferenced environments. Journal of Intelligent \& Robotic Systems, 83(3-4):635-648, 2016.

[3] Hamed Ketabdar, Peyman Moghadam, and Mehran Roshandel. Pingu: A new miniature wearable device for ubiquitous computing environments. In Sixth International Conference on Complex, Intelligent, and Software Intensive Systems, pages 502-506, 2012. 
[4] Lokesh Dhakar, Huicong Liu, FEH Tay, and Chengkuo Lee. A new energy harvester design for high power output at low frequencies. Sensors and Actuators A: Physical, 199:344-352, 2013.

[5] W. Y. Toh, Y. K. Tan, W. S. Koh, and L. Siek. Autonomous wearable sensor nodes with flexible energy harvesting. IEEE Sensors Journal, 14(7):2299-2306, July 2014.

[6] Achraf Ben Amar, Ammar B Kouki, and Hung Cao. Power approaches for implantable medical devices. Sensors, 15(11):28889-28914, 2015.

[7] Stephen Vidas and Peyman Moghadam. HeatWave: a handheld 3D thermography system for energy auditing. Energy and Buildings, 66:445-460, November 2013.

[8] Stephen Vidas, Peyman Moghadam, and Sridha Sridharan. Real-time mobile 3D temperature mapping. IEEE Sensors Journal, 15(2):1145-1152, 2014.

[9] Maisam Wahbah, Mohammad Alhawari, Baker Mohammad, Hani Saleh, and Mohammed Ismail. Characterization of human body-based thermal and vibration energy harvesting for wearable devices. IEEE Journal on Emerging and Selected Topics in Circuits and Systems, 4(3):354-363, 2014.

[10] Paul D Mitcheson. Energy harvesting for human wearable and implantable bio-sensors. In 2010 Annual International Conference of the IEEE Engineering in Medicine and Biology, pages 3432-3436. IEEE, 2010.

[11] Shad Roundy, Paul K Wright, and Jan Rabaey. A study of low level vibrations as a power source for wireless sensor nodes. Computer communications, 26(11):1131-1144, 2003.

[12] Q. Brogan, T. O'Connor, and D. S. Ha. Solar and thermal energy harvesting with a wearable jacket. In 2014 IEEE International Symposium on Circuits and Systems (ISCAS), pages 1412-1415, June 2014.

[13] Michele Magno, Davide Brunelli, Lukas Sigrist, Renzo Andri, Lukas Cavigelli, Andres Gomez, and Luca Benini. Infinitime: Multi-sensor wearable bracelet with human body harvesting. Sustainable Computing: Informatics and Systems, 11:38 - 49, 2016. SI: \{IGCC 2014.

[14] Mahammad A Hannan, Saad Mutashar, Salina A Samad, and Aini Hussain. Energy harvesting for the implantable biomedical devices: issues and challenges. Biomedical engineering online, 13(1):1, 2014.

[15] Y Minami and E Nakamachi. Development of enhanced piezoelectric energy harvester induced by human motion. In 2012 Annual International Conference of the IEEE Engineering in Medicine and Biology Society, pages 1627-1630. IEEE, 2012.

[16] Vinod R Challa, MG Prasad, Yong Shi, and Frank T Fisher. A vibration energy harvesting device with bidirectional resonance frequency tunability. Smart Materials and Structures, 17(1):015035, 2008.

[17] Emilio Sardini and Mauro Serpelloni. An efficient electromagnetic power harvesting device for lowfrequency applications. Sensors and Actuators A: Physical, 172(2):475-482, 2011.

[18] Bin Yang, Chengkuo Lee, Wenfeng Xiang, Jin Xie, Johnny Han He, Rama Krishna Kotlanka, Siew Ping Low, and Hanhua Feng. Electromagnetic energy harvesting from vibrations of multiple frequencies. Journal of Micromechanics and Microengineering, 19(3):035001, 2009.

[19] Yulong Zhang, Tianyang Wang, Anxin Luo, Yushen Hu, Xinxin Li, and Fei Wang. Micro electrostatic energy harvester with both broad bandwidth and high normalized power density. Applied energy, 212:362371, 2018.

[20] Chongfeng Wei and Xingjian Jing. A comprehensive review on vibration energy harvesting: Modelling and realization. Renewable and Sustainable Energy Reviews, 74:1-18, 2017.

[21] Tanju Yildirim, Mergen H Ghayesh, Weihua Li, and Gursel Alici. A review on performance enhancement techniques for ambient vibration energy harvesters. Renewable and Sustainable Energy Reviews, 71:435449, 2017.

[22] Graeme Best, Peyman Moghadam, Navinda Kottege, and Lindsay Kleeman. Terrain classification using a hexapod robot. In Proceedings of the Australasian Conference on Robotics and Automation, 2013.

[23] N. Kottege, C. Parkinson, P. Moghadam, A. Elfes, and S.P.N. Singh. Energetics-informed hexapod gait transitions across terrains. In IEEE International Conference on Robotics and Automation (ICRA '15), pages 5140-5147, May 2015.

[24] Chanoh Park, Peyman Moghadam, Soohwan Kim, Alberto Elfes, Clinton Fookes, and Sridha Sridharan. Elastic LiDAR fusion: Dense map-centric continuous-time slam. In IEEE International Conference on Robotics and Automation (ICRA), pages 1206-1213, 2018.

[25] Raul Morais, Nuno M. Silva, Paulo M. Santos, Clara M. Frias, and Jorge A.F. Ferreira. Double permanent magnet vibration power generator for smart hip prosthesis. ASME Transactions on Mechatronics, pages 259-268, 2011.

[26] Alireza Khaligh, Peng Zeng, and Chong Zheng. Kinetic energy harvesting using piezoelectric and electromagnetic technologies - state of the art. IEEE Transactions on Industrial Electronics, 57:850-860, 2010.

[27] BL Ooi and JM Gilbert. Design of wideband vibration-based electromagnetic generator by means of dual-resonator. Sensors and Actuators A: Physical, 213:9-18, 2014.

[28] Xiudong Tang and Lei Zuo. Enhanced vibration energy harvesting using dual-mass systems. Journal of sound and vibration, 330(21):5199-5209, 2011. 
[29] SM Shahruz. Design of mechanical band-pass filters for energy scavenging: multi-degree-of-freedom models. Journal of Vibration and Control, 14(5):753-768, 2008.

[30] J. Kymissis, Paradiso J. Kendall, C., and N. Gershenfeld. Parasitic power harvesting in shoes. Proc. 2nd IEEE Int. Conf. Wearable Comput., 2:132-139, 1998.

[31] José Gerardo Rocha, Luis Miguel Goncalves, PF Rocha, MP Silva, and Senentxu Lanceros-Mendez. Energy harvesting from piezoelectric materials fully integrated in footwear. IEEE transactions on industrial electronics, 57(3):813-819, 2010.

[32] Farbod Khameneifar, Siamak Arzanpour, and Mehrdad Moallem. A piezoelectric energy harvester for rotary motion applications: Design and experiments. ASME Transactions on Mechatronics, 18:1527-1534, October 2013.

[33] C. Shu, Y. and C. Lien, I. Analysis of power output for piezoelectric energy harvesting systems. SmartMater. Struct., 15:1499-1512, Dec. 2006.

[34] BP Mann and ND Sims. Energy harvesting from the nonlinear oscillations of magnetic levitation. Journal of Sound and Vibration, 319(1):515-530, 2009.

[35] Abdullah Nammari, Seth Doughty, Dustin Savage, Leland Weiss, Arun Jaganathan, and Hamzeh Bardaweel. Broadband magnetic levitation-based nonlinear energy harvester. In SPIE Commercial+ Scientific Sensing and Imaging, pages 98650L-98650L. International Society for Optics and Photonics, 2016.

[36] Hong-yan Wang, Li-hua Tang, Yuan Guo, Xiao-biao Shan, and Tao Xie. A 2dof hybrid energy harvester based on combined piezoelectric and electromagnetic conversion mechanisms. Journal of Zhejiang University-SCIENCE A (Applied Physics and Engineering), 15:711-722, 2014. 